The outcome of this competition depends in part on the intensity of the original training and the number of extinction trials; if the original training is highly robust and/or the number of extinction trials is too small, the "inhibitory" or CS-noUS trace may not gain appreciable control of behavior. Our findings, using two different species, different memory paradigms, and different consolidation blockers with different molecular targets, suggest that the trace that retains or is in the process of gaining appreciable control over behavior after the retrieval session (i.e., becomes dominant) is the one that displays transient sensitivity to the consolidation blocker. In other words, the stability of the trace, as judged by insensitivity to consolidation blockers, is inversely correlated with trace dominance. It remains possible that the drugs block extinction under some conditions but accelerate it under other conditions. This differential effect is highly unlikely, particularly in light of the aforementioned prevalent view that extinction is relearning $(2,18-20)$ and that consolidation blockers disrupt the formation of the long-term trace (21-23); acceleration of extinction by the two different consolidation blockers might have implied that these agents enhance memory formation, for which there is no evidence.

Our findings could be construed in the context of the reconsolidation hypothesis, which posits that after retrieval the activated trace must undergo a process of stabilization to enter again into a long-term phase (7-10). A caveat is, however, appropriate. We do not yet know whether the effect of consolidation blockers after retrieval is identical to their effect after acquisition. The possibility that the effect might be different is hinted at by reports that molecular and circuit mechanisms that subserve the acquisition of original and extinguishing traces share components but are nonidentical $(4,5,15)$. For example, although we did not detect spontaneous recovery of the blocked memory [tested at 8 days in rat CTA and 2 days in medaka fear conditioning (24)], we cannot exclude the possibility that other manipulations might unveil a latent, depressed trace. This could indicate performance or retrieval deficit rather than storage deficit. It is noteworthy that although a predominant assumption is that blockade of consolidation after acquisition leads to storage deficit, explanations based on retrieval deficits were not abandoned [e.g., (11)].

The proposed relationship between the susceptibility to disruption and the dominance of the trace after retrieval, which probably is an experimental manifestation of the theoretical dichotomy "active-inactive" memory (25), may explain some lingering discrep- ancies in the literature on the effect of consolidation blockers after retrieval. It may also be of potential value in designing protocols for selective modification of different longterm associations of a target item in memory.

\section{References and Notes}

1. I. P. Pavlov, Conditioned Reflexes: An Investigation of the Physiological Activity of the Cerebral Cortex (Oxford Univ. Press, London, 1927).

2. Y. Dudai, Memory from A to Z: Keywords, Concepts, and Beyond (Oxford Univ. Press, Oxford, 2002)

3. J. Bures, F. Bermudez-Rattoni, T. Yamamoto, Conditioned Taste Aversion: Memory of a Special Kind (Oxford Univ. Press, New York, 1998).

4. D. E. Berman, Y. Dudai, Science 291, 2417 (2001)

5. A. Bahar, A. Samuel, S. Hazvi, Y. Dudai, Eur. J. Neurosci. 17, 1527 (2003).

6. M. R. M. Vianna, G. Szapiro, J. L. McGaugh, J. H. Medina, I. Izquierdo, Proc. Natl. Acad. Sci. U.S.A. 98, 12251 (2001).

7. J. R. Misanin, R. R. Miller, D. J. Lewis, Science 160,554 (1968).

8. S. J. Sara, Learn. Mem. 7, 73 (2000).

9. K. Nader, G. E. Schafe, J. E. LeDoux, Nature 406, 722 (2000).

10. S. M. Taubenfeld, M. H. Milekic, B. Monti, C. M. Alberini, Nature Neurosci. 4, 813 (2001).

11. P. M. Millin, E. W. Moody, D. C. Riccio, Nature Rev. Neurosci. 2, 68 (2001).

12. K. V. Anokhin, A. A. Tiunova, S. P. R. Rose, Eur. J. Neurosci. 15, 1759 (2002).
13. K. Nader, Trends Neurosci. 26, 65 (2003).

14. Information on materials and methods is available on Science Online.

15. D. E. Berman, S. Hazvi, J. Stehberg, A. Bahar, Y. Dudai, Learn. Mem. 10, 16 (2003).

16. G. E. Savage, in The Central Nervous System and Fish Behavior, D. Ingle, Ed. (Univ. of Chicago Press, Chicago, 1968), pp. 127-138.

17. T. Yamamoto, Medaka (Killifish): Biology and Strains (Keigaku, Tokyo, 1975).

18. R. A. Rescorla, Q. J. Exp. Psychol. 49B, 245 (1996).

19. M. E. Bouton, J. Exp. Psychol. Anim. Behav. Proc. 20, 219 (1994).

20. K. M. Myers, M. Davis, Neuron 36, 567 (2002).

21. H. P. Davis, L. R. Squire, Psychol. Bull. 96, 518 (1984).

22. P. G. Montarolo et al., Science 234, 1249 (1986).

23. Y. Dudai, R. G. M. Morris, in Brain, Perception, Memory: Advances in Cognitive Sciences, J. J. Boluhis, Ed. (Oxford Univ. Press, Oxford, 2000), pp. 149-162.

24. M. Eisenberg, T. Kobilo, Y. Dudai, data not shown.

25. D. J. Lewis, Psychol. Bull. 86, 1054 (1979).

26. We thank A. Bahar, A. Desmedt, N. Dorfman, and J. Stehberg for valuable discussions, and M. Katkov for assistance in programming. Supported by the Human Frontier Science Program, the Israel Science Foundation, and the Volkswagen Stiftung.

Supporting Online Material

www.sciencemag.org/cgi/content/full/301/5636/1102/ DC1

Materials and Methods

19 May 2003; accepted 10 July 2003

\title{
Encoding Predictive Reward Value in Human Amygdala and Orbitofrontal Cortex
}

\author{
Jay A. Gottfried,* John O'Doherty, Raymond J. Dolan
}

\begin{abstract}
Adaptive behavior is optimized in organisms that maintain flexible representations of the value of sensory-predictive cues. To identify central representations of predictive reward value in humans, we used reinforcer devaluation while measuring neural activity with functional magnetic resonance imaging. We presented two arbitrary visual stimuli, both before and after olfactory devaluation, in a paradigm of appetitive conditioning. In amygdala and orbitofrontal cortex, responses evoked by a predictive target stimulus were decreased after devaluation, whereas responses to the nondevalued stimulus were maintained. Thus, differential activity in amygdala and orbitofrontal cortex encodes the current value of reward representations accessible to predictive cues.
\end{abstract}

An organism's ability to predict future events, such as food or danger, on the basis of relevant sensory cues is emblematic of associative learning. This phenomenon can be studied with classical conditioning, whereby a previously neutral item (the conditioned stimulus, $\mathrm{CS}+$ ) acquires importance after being paired with a biologically salient reinforcer (the unconditioned stimulus, UCS). The efficacy of conditioning depends on establishing CS-UCS links, but evidence suggests

Functional Imaging Laboratory, Wellcome Department of Imaging Neuroscience, 12 Queen Square, London WC1N 3BG, UK.

*To whom correspondence should be addressed. Email: j.gottfried@fil.ion.ucl.ac.uk that a CS + can invoke multiple, unique UCS representations, including sensory properties, reward value, or associated affective states (1). Clarifying the neural substrates that support these associative links has important implications for biological models of reinforcement learning $(2,3)$.

Neuroimaging studies emphasize the roles of amygdala and orbitofrontal cortex (OFC) in human classical conditioning (4-7), but no experiment has characterized the psychological underpinnings of these activations. Reinforcer devaluation offers a means of dissociating among the various central representations that a $\mathrm{CS}+$ may engage. This approach has been applied to animal studies of appetitive learning, which show that damage to amygdala and OFC 
interferes with the effects of reinforcer devaluation $(8-11)$. However, it remains unclear from lesion studies what precise information is encoded within these structures.

We used functional magnetic resonance imaging (fMRI) to determine the impact of reinforcer devaluation on responses evoked by predictive cues. If amygdala and OFC maintain representations of predictive reward value, then neural responses evoked by a $\mathrm{CS}+$ should be sensitive to experimental manipulations that devalue predicted reward. On the other hand, insensitivity to devaluation would indicate that the role of these areas in associative learning is independent of, or precedes linkage to, central representations of their reward value. Thirteen hungry subjects were scanned during learning and anticipation of two food-based olfactory rewards, both before and after selective satiation (12). One odor was destined for reinforcer devaluation (target UCS), whereas the other underwent no motivational shift (nontarget UCS). Arbitrary visual images comprised target and nontarget CS + stimuli, which were either paired $(\mathrm{CS}+\mathrm{p})$ with the corresponding UCS or unpaired $(\mathrm{CS}+\mathrm{u})(4)$. Another image was never paired with odor (CS-) (Fig. 1).

During initial training, subjects learned the picture-odor associations while performing a visuospatial discrimination task. Reaction times (RTs) provided independent evidence for conditioning $(6,13)$ and confirmed that subjects responded significantly faster to the target and nontarget $\mathrm{CS}+$, compared with CS-, during the first half-session (Fig. 2A). Significant neural responses in posterior amygdala, rostromedial OFC, ventral midbrain, primary olfactory (piriform) cortex, insula, and hypothalamus (Fig. 2B and table S1) highlighted regions that participate in the acquisition of picture-odor contingencies. This network is similar to activation patterns evoked in previous imaging studies of appetitive conditioning $(6,7)$.

After training, subjects received the same contingencies in two further sessions. Between sessions, subjects were removed from the scanner and fed until sated with a meal corresponding to the target odor identity, thereby reducing the motivational value of the target UCS without affecting nontarget UCS value. Hunger level and food pleasantness both significantly declined at the end of the meal (12) (Fig. 3, A and B). Subjects reported no significant differences in odor pleasantness between target (4.42 \pm 0.85 ; mean \pm SEM) and nontarget $(4.21 \pm 1.11) \mathrm{UCS}$ in the pre-satiety state $(P=0.75)$. Critically, ratings from pre- to post-feeding significantly decreased for the target UCS $(P<0.01)$ in the absence of changes for the nontarget UCS $(P=0.46)$, reflecting the efficacy of selective satiation in lessening the value of the target UCS
(Fig. 3C). Ratings of odor intensity did not differ between target and nontarget UCS or as an effect of satiety. There were no significant differences in sniff amplitude or latency between target and nontarget conditions in pre- or post-satiety sessions.

Our aim was to identify brain regions showing differential responses to the target $\mathrm{CS}+\mathrm{u}$ from pre- to post-satiety (relative to nontarget $\mathrm{CS}+\mathrm{u}$ responses) (12). We found significant response decrements in left dorsomedial amygdala, which spanned regions adjacent to posterior cortical and basomedial amygdala nuclei (Fig. 3D). Significant differential activity was also detected in multiple areas of OFC (Fig. 3E). Contrast estimates of signal change from amygdala and OFC demonstrated satiety-related declines in target $\mathrm{CS}+\mathrm{u}$ activity with preserved nontarget $\mathrm{CS}+\mathrm{u}$ activity (Fig. 3, D and E), paralleling behavioral effects of satiation. Conversely, satiety-sensitive neural responses in ventral striatum, insula, and anterior cingulate exhibited a different pattern of activity, reflecting both decreases to the target $\mathrm{CS}+\mathrm{u}$ and increases to the nontarget $\mathrm{CS}+\mathrm{u}$ (Fig. $3 \mathrm{~F}$ and table $\mathrm{S} 2$ ).
Numerous studies have documented the effect of hunger states on food-related processing in OFC, amygdala, and insula (1418), but none has investigated the influence of selective satiation on evoked activity patterns in the context of associative learning. Our results underscore the selective impact of hunger and satiety on the neural correlates of reward prediction: When a food changes from delectable to distasteful, the brain responses evoked by a predictive cue are attenuated in areas that maintain responses to predictors of other palatable stimuli. Thus, amygdala and OFC activity evoked by the target $\mathrm{CS}+\mathrm{u}$ decreased from pre- to post-satiety, parallel to the reward value of the target UCS, whereas activity did not change to the nontarget $\mathrm{CS}+\mathrm{u}$, indicating that the concurrent reward value of predictive stimuli may be represented in amygdala and OFC. This proposal accords with animal studies of reinforcer devaluation $(8-11)$ and olfactory reversal learning $(19,20)$, demonstrating the importance of amygdala-OFC networks to motivational and incentive processes.

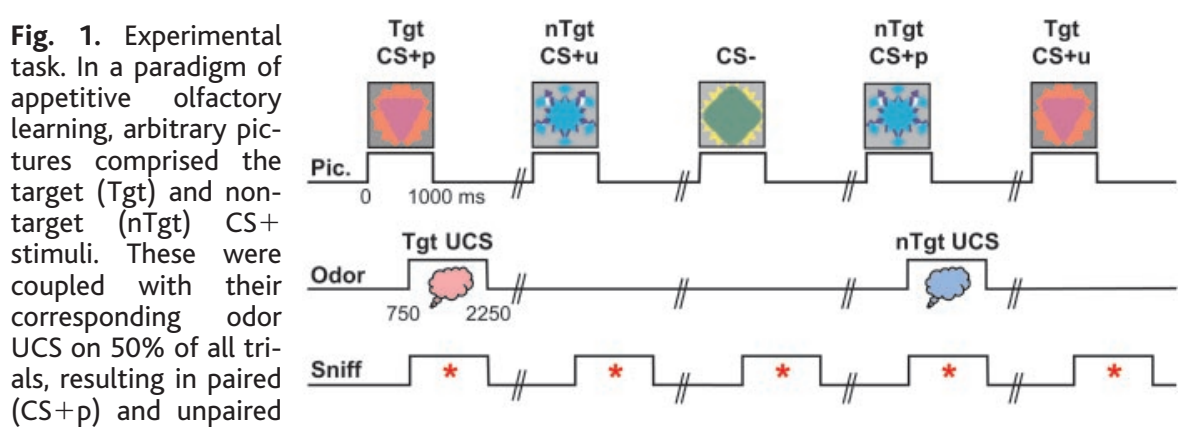

$(\mathrm{CS}+\mathrm{p})$ and unpaired

$(\mathrm{CS}+\mathrm{u})$ event types. A nonconditioned stimulus (CS-) was never paired with odor. During each trial, subjects indicated whether the picture appeared on the left or right side, and they sniffed upon delivery of a sniff cue (red asterisk). The same contingencies and task were repeated for all sessions.

Fig. 2. (A) Mean RTs pro- A vided an objective index of learning. Subjects responded significantly faster to the CS+ stimuli than to the CS- in the first half of training. Error bars in all figures reflect SEM. *, $P<$ 0.05 . (B to D) Neural responses detected in a random-effects group analysis of olfactory learning (training session). Significant incremental activations were evoked by the CS+u stimuli (relative to $\mathrm{CS}_{-}$) in amygdala (amy), orbitofrontal cortex (ofc), piriform cortex (pir), insula (ins), and ventral midbrain (vmb). Statistical maps (threshold, $P<0.001$ ) are overlaid on coronal (B), sagittal (C), and axial (D) sections from the mean T1-weighted structural scan. See table S1 for activation peaks.

C
B
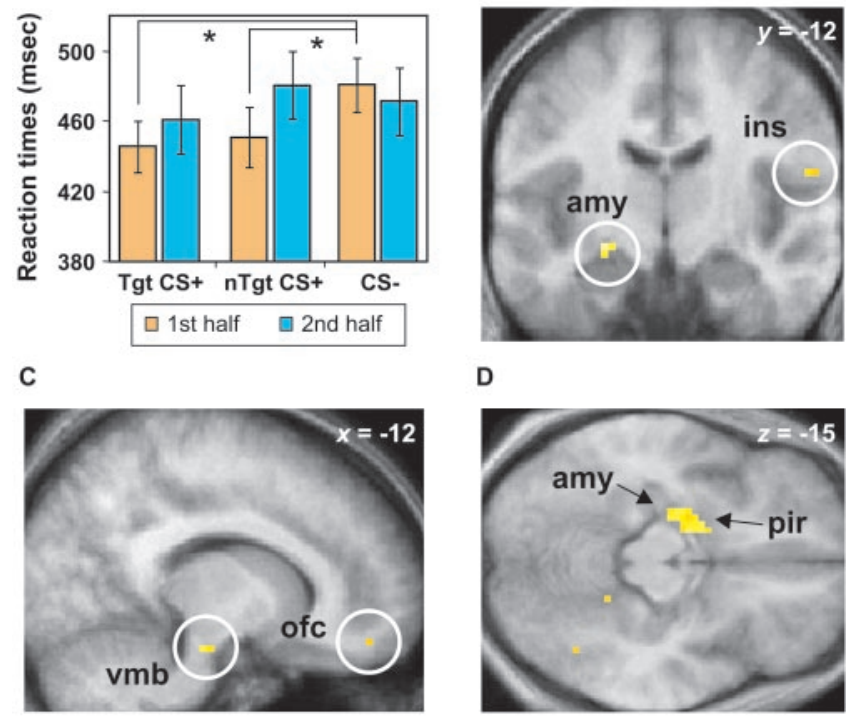
In contrast, other brain regions, including ventral striatum, insula, and cingulate cortex, exhibited both response decreases to the target $\mathrm{CS}+\mathrm{u}$ and response increases to the nontarget $\mathrm{CS}+\mathrm{u}$. As an effect of selective satiation, the relative reward value of the nontarget (nondevalued) $\mathrm{CS}+\mathrm{u}$ might be enhanced. Human physiological studies of satiety indicate that the pleasantness of an unsated food can actually increase at the same time that sated items become less appetizing (21). Indeed, shifting the balance of reward representation from consumed to unconsumed foods may optimize the motivational basis for food selection.

Do the satiety-related effects we observed reflect an ability of a $\mathrm{CS}+$ to access representations of UCS reward value? We determined whether activations modulated by reinforcer devaluation are expressed in the same regions that encode representations of odor UCS (12). Significant overlapping responses in dorsal amygdala and caudal OFC (table S2) suggest that the $\mathrm{CS}+$ gains access to UCS representations of reward value encoded in these areas.
Fig. 3. (A to C) Mean behavioral ratings of hunger level $(A)$, food pleasantness $(B)$, and odor pleasantness (C) illustrating selective devaluation of the target UCS. (D to F) Satiety-sensitive neural activations that paralleled the behavioral effect. (D) In dorsomedial amygdala (at $x=-15, y$ $=-6, z=-18)$, neural responses elicited by the target $\mathrm{CS}+\mathrm{u}$ declined from pre- to post-satiety, whereas nontarget $\mathrm{CS}+\mathrm{u}$ activity was unchanged. The middle panel is magnified from the left. Activations are superimposed on coronal sections $(P<0.01$ for display). On the right, amygdala signal change is plotted as contrasts of parameter estimates (betas) for both target and nontarget $\mathrm{CS}+\mathrm{u}$, after adjusting for CSbaselines. (E) In OFC (at $24,33,-12$ ), a similar activation pattern was observed. Neural responses are displayed in axial and coronal formats. (F) By comparison, significant
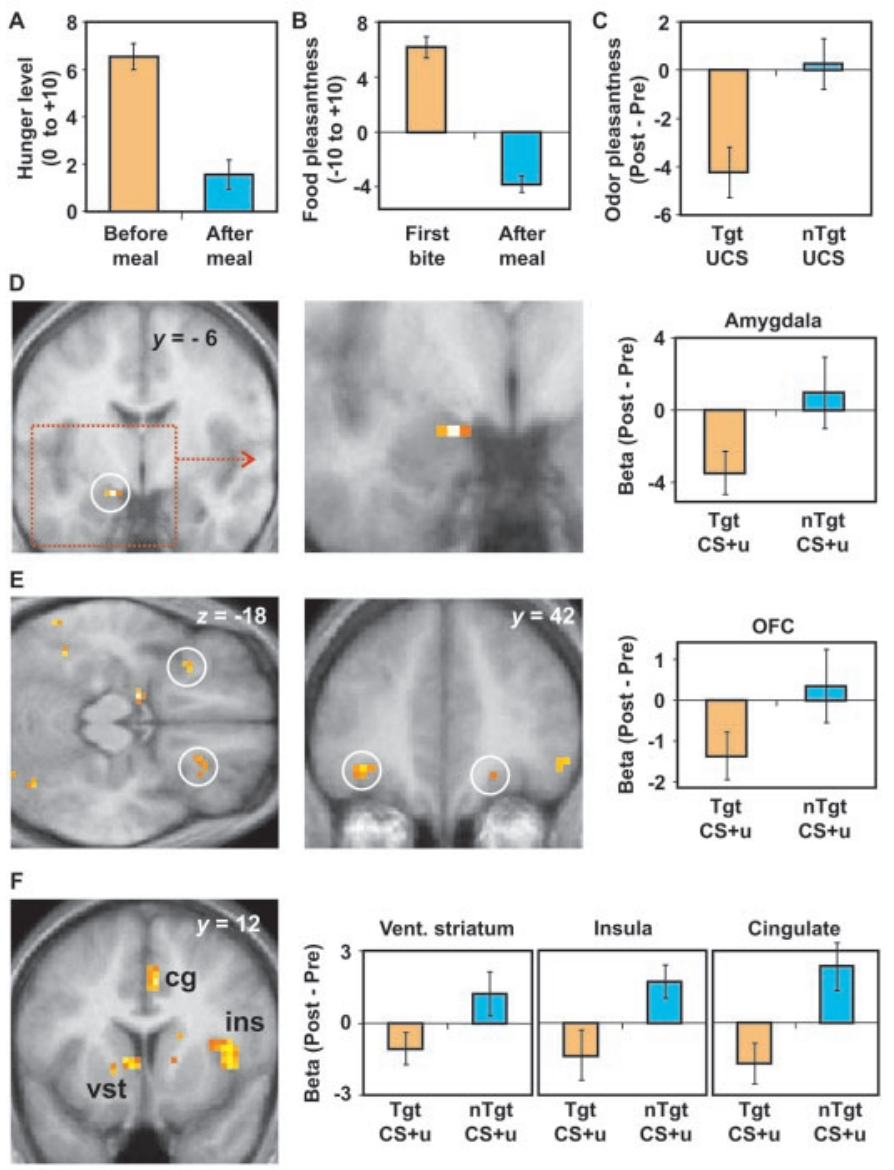

satiety-related effects in ventral striatum (vst), insula (ins), and anterior cingulate (cg) also reflected response increases to the nontarget CS $+u$. Peak coordinates are listed in table S2.

Fig. 4. Location of satietyrelated responses that are a subset of those evoked during olfactory learning. Significant activations $(P<0.001)$ in amygdala (amy), piriform cortex (pir), and OFC are depicted in axial orientation (left), with serial cross sections where indicated along the coronal axis (right). See table S3 for peak activation coordinates.
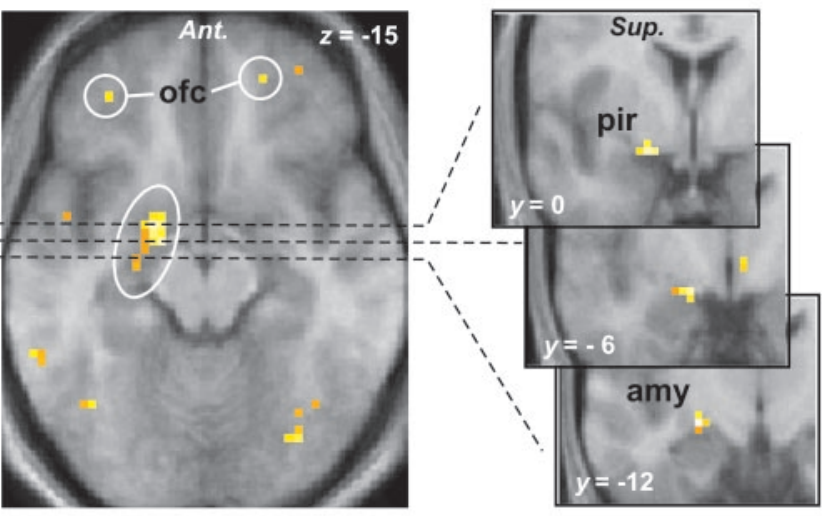

Satiety-sensitive regions not detected in this analysis (Fig. 3F) are also critical to associative learning but appear to mediate different aspects of reward prediction (1, $22)$. These structures could help assign reward value to the $\mathrm{CS}+$ itself or discriminate relative values among predictive stimuli.

Finally, we examined whether brain regions that encode predictive reward value also participate in the initial acquisition of stimulus-reward contingencies (12). We detected significant responses in medial temporal lobe, with peaks in amygdala posteriorly and piriform cortex anteriorly (Fig. 4). Responses were also observed in OFC, insula, hypothalamus, and cingulate cortex (table S3), indicating that many of the brain regions maintaining representations of predictive reward may be a subset of those engaged in associative learning. Animal (23) and human $(6,24)$ studies indicate that primary olfactory structures are not mere sensory relays, but participate in higher order computations related to learning and motivation. The same considerations can be applied to taste processing: The anterior portion of insula activated here falls within a zone defined as human gustatory cortex (25), suggesting that aspects of food-based reward are also updated in these structures.

In animal studies of devaluation, behavior is typically assessed with procedures uncontaminated by new learning $(8-11)$. In our design, because picture-odor pairs were repeatedly presented after devaluation, it is possible that the satiety-specific effects could reflect new associative learning between the target $\mathrm{CS}+$ and the devalued UCS. Consequently, we performed a supplementary analysis modeling condition by time interactions for the pre- and postsatiety phases. Satiety-related decrements (of mean target $\mathrm{CS}+\mathrm{u}$ activity) were still detected in identical regions of amygdala and $\mathrm{OFC}$, but there was no significant timedependent (learning-related) response decline in these brain areas (26). Nevertheless, given the rapidity of initial learning (during the training phase), the possibility remains that new learning could have taken place even faster and contributed to the satiety-related responses described here.

Patients with damage to medial temporal and basal frontal lobes commonly engage in maladaptive behaviors, tending to choose immediate rewards without regard for future consequences (27). Defective encoding of (or access to) updated reward value in amygdala and OFC could explain the inability of such patients to modify responses when expected outcomes change (20). Our findings also have implications for the feeding derangements described in Kluver-Bucy syndrome (28) and frontotemporal dementia (29). These patients display symptoms ranging from in- 
creased appetite and changes in food preference to hyperorality and consumption of nonfoods. Such phenomena may arise out of a disabled network involving OFC and amygdala, whereby routine (learned) food cues no longer recruit motivationally appropriate representations of food-based reward value.

Theoretical and computational models of reward learning postulate the concept of motivational "gates" that traffic information flow between internal representations of the $\mathrm{CS}+$ and the $\operatorname{UCS}(2,30)$. These gates are the targets of motivational signals (likely a combination of sensory, visceral, autonomic, and interoceptive factors) and determine the likelihood that stimulus-reward associations activate appetitive systems. The neural mechanisms that support these processes are not well characterized. Our data show that neural responses evoked by a $\mathrm{CS}+$ in amygdala, OFC, ventral striatum, insula, cingulate, and hypothalamus are directly modulated by hunger states, indicating that this structural network underpins Pavlovian incentive behavior in a manner that meets the requirements of a motivational gate.

\section{References and Notes}

1. R. N. Cardinal, J. A. Parkinson, J. Hall, B. J. Everitt, Neurosci. Biobehav. Rev. 26, 321 (2002).

2. P. Dayan, B. W. Balleine, Neuron 36, 285 (2002)

3. P. R. Montague, G. S. Berns, Neuron 36, 265 (2002).

4. C. Buchel, J. Morris, R. J. Dolan, K. J. Friston, Neuron 20, 947 (1998).

5. K. S. LaBar, J. C. Gatenby, J. C. Gore, J. E. LeDoux, E. A. Phelps, Neuron 20, 937 (1998).

6. J. A. Gottfried, J. O'Doherty, R. J. Dolan, J. Neurosci. 22, 10829 (2002)

7. J. O'Doherty, R. Deichmann, H. D. Critchley, R. J. Dolan, Neuron 33, 815 (2002).

8. T. Hatfield, J. S. Han, M. Conley, M. Gallagher, P. Holland, J. Neurosci. 16, 5256 (1996).

9. L. Malkova, D. Gaffan, E. A. Murray, J. Neurosci. 17, 6011 (1997).

10. M. Gallagher, R. W. McMahan, G. Schoenbaum, J. Neurosci. 19, 6610 (1999)

11. M. G. Baxter, A. Parker, C. C. Lindner, A. D. Izquierdo, E. A. Murray, J. Neurosci. 20, 4311 (2000).

12. Materials and methods are available as supporting material on Science Online.

13. H. D. Critchley, C. J. Mathias, R. J. Dolan, Neuron 33, 653 (2002).

14. H. D. Critchley, E. T. Rolls, J. Neurophysiol. 75, 1673 (1996).

15. B. W. Balleine, A. Dickinson, J. Neurosci. 20, 8954 (2000).

16. J. O'Doherty et al., Neuroreport 11, 893 (2000).

17. K. S. LaBar et al., Behav. Neurosci. 115, 493 (2001).

18. D. M. Small, R. J. Zatorre, A. Dagher, A. C. Evans, M. Jones-Gotman, Brain 124, 1720 (2001).

19. E. T. Rolls, H. D. Critchley, R. Mason, E. A. Wakeman, J. Neurophysiol. 75, 1970 (1996).

20. G. Schoenbaum, A. A. Chiba, M. Gallagher, J. Neurosci. 19, 1876 (1999)

21. B. J. Rolls, E. T. Rolls, E. A. Rowe, K. Sweeney, Physiol. Behav. 27, 137 (1981).

22. W. Schultz, L. Tremblay, J. R. Hollerman, Cereb. Cortex 10, 272 (2000).

23. G. Schoenbaum, H. Eichenbaum, J. Neurophysiol. 74, 733 (1995).

24. J. A. Gottfried, R. Deichmann, J. S. Winston, R. J. Dolan, J. Neurosci. 22, 10819 (2002).

25. D. M. Small et al., Neuroreport 10, 7 (1999).

26. Data not shown

27. A. Bechara, A. R. Damasio, H. Damasio, S. W. Anderson, Cognition 50, 7 (1994).
28. H. Terzian, G. Dalle Ore, Neurology 5, 373 (1955).

29. M. Ikeda, J. Brown, A. J. Holland, R. Fukuhara, J. R. Hodges, J. Neurol. Neurosurg. Psychiatry 73, 371 (2002).

30. A. Dickinson, B. Balleine, in Learning, Motivation, and Emotion, C. R. Gallistel, Ed., volume 3 of Stevens' Handbook in Experimental Psychology, 3rd ed. (Wiley, New York, 2002).

31. The authors thank J. M. Kilner and J. S. Winston for helpful comments. This work was supported by a Howard Hughes
Medical Institute Physician-Postdoctoral Fellowship Grant (J.A.G.) and a Wellcome Trust Programme Grant (R.J.D.). Supporting Online Material www.sciencemag.org/cgi/content/full/301/5636/1104/ DC1

Materials and Methods

Tables S1 to S3

References

11 June 2003; accepted 14 July 2003

\title{
SIR1, an Upstream Component in Auxin Signaling Identified by Chemical Genetics
}

\author{
Yunde Zhao, ${ }^{1 *}$ Xinhua Dai, ${ }^{1}$ Helen E. Blackwell, ${ }^{2} \dagger$ \\ Stuart L. Schreiber, ${ }^{2}$ Joanne Chory ${ }^{3}$
}

\begin{abstract}
Auxin is a plant hormone that regulates many aspects of plant growth and development. We used a chemical genetics approach to identify SIR1, a regulator of many auxin-inducible genes. The sir 1 mutant was resistant to sirtinol, a small molecule that activates many auxin-inducible genes and promotes auxin-related developmental phenotypes. SIR1 is predicted to encode a protein composed of a ubiquitin-activating enzyme E1-like domain and a Rhodanese-like domain homologous to that of prolyl isomerase. We suggest a molecular context for how the auxin signal is propagated to exert its biological effects.
\end{abstract}

Auxin has been implicated in almost every aspect of plant growth and development. Molecular genetics studies on auxin-resistant Arabidopsis mutants $(1,2)$ and biochemical analyses of early auxin-inducible genes $(3,4)$ have elucidated many aspects of auxin signaling. The current model of auxin signaling suggests that negative regulators such as the auxin/indole-3acetic acid (Aux/IAA) proteins are targeted for degradation in an auxin-dependent manner through the ubiquitin-related protein degradation machinery, thereby derepressing a network of genes to guide proper growth and development (5).

Analysis of auxin signaling by classical genetics is complicated by auxin polar transport, a process whereby an auxin-concentration gradient is maintained among neighboring cells (6). This process limits the accessibility of certain cells and tissues to exogenous auxin, and therefore certain auxin signaling components might well be missed from genetic screens for mutants that are resistant to exogenous auxin.

${ }^{1}$ Section of Cell and Developmental Biology, Division of Biological Sciences, University of California at San Diego, 9500 Gilman Drive, La Jolla, CA 92093-0116, USA. 'Department of Chemistry and Chemical Biology, Howard Hughes Medical Institute (HHMI), Harvard University, 12 Oxford Street, Cambridge, MA 02138, USA. ${ }^{3}$ The Plant Biology Laboratory, HHMI and The Salk Institute, 10010 North Torrey Pines Road, La Jolla, CA 92037, USA.

*To whom correspondence should be addressed. Email: yzhao@biomail.ucsd.edu

$\dagger$ Present address: Department of Chemistry, University of Wisconsin-Madison, 1101 University Avenue, Madison, WI 53706-1396, USA.
Moreover, auxin polar transport and auxin signaling are well-coordinated, interdependent processes, making it difficult to isolate and specifically analyze either process. Here we identify a tool to modulate auxin signaling exclusively, without affecting auxin polar transport, in order to analyze the molecular mechanisms of auxin signaling.

We used a chemical genetics (7) approach to identify an auxin signal transduction component in Arabidopsis. Sirtinol (Fig. 1A), an inhibitor of the Sirtuin family of nicotinamide adenine dinucleotide (NAD)-dependent deacetylases in Saccharomyces cerevisiae, affects root and vascular tissue development in Arabidopsis (8). Sirtinol specifically activates many auxin-inducible genes, thereby promoting several auxin-related developmental phenotypes. With this approach, we identified the gene SIRI as a key regulator of many auxininducible genes.

We screened for compounds that could alter the expression pattern and/or levels of an auxin reporter line, DR5-GUS (9). Sirtinol caused up-regulation and ectopic expression of the auxin reporter gene (10) that was induced throughout the plant, with the highest expression levels along the vascular tissues (Fig. 1B). We next performed a microarray analysis using the Arabidopsis "whole genome" chip (Affymetrix) to determine whether sirtinol activated the expression of auxin-inducible genes at the transcriptional level (10). Of the 23,000 genes on the chip, about 16,000 genes gave signals that were significantly above background levels in all samples. Sirtinol treatment 